\title{
Effusive-constrictive pericarditis: current perspectives
}

This article was published in the following Dove Press journal: Journal of Vascular Diagnostics and Interventions

\author{
Bernhard Maisch \\ Faculty of Medicine, Philipps- \\ University Marburg and Center for \\ Heart and Vessels, Marburg, Germany
}

\begin{abstract}
Effusive-constrictive pericarditis (ECP) is defined by concurrent pericardial effusion and pericardial constriction. Whereas the pericardial cavity is typically obliterated in patients with constrictive pericarditis without any effusion, in patients with ECP the scarred pericardium not only constricts the cardiac volume but can also put pericardial fluid under increased pressure, leading to signs suggestive of cardiac tamponade. These hemodynamic features can persist even after the pericardial effusion is removed. The underlying pathogenetic process predominantly involves the visceral pericardium or epicardium. It combines visceral pericardial constriction with pericardial inflammation. Its etiology may be as variable as the causes of effusive or constrictive pericardial disease, which may be infective (e.g., bacterial or viral), malignant or autoreactive. The label idiopathic ECP should be avoided and restricted to only those cases in whom, after thorough clinical and pathological workup including PCR for microbial agents in pericardial fluid and cardiac tissue from peri- and epicardial or endomyocardial biopsies and the assessment of autoimmune processes, no definite diagnosis can be made. Since a considerable number of ECP patients have an inflammatory and reversible pericardial reaction, they can improve under causal and/or anti-inflammatory treatment. The treatment should therefore be based first on eradicating the underlying etiological factors. If such a treatment course remains ineffective, pericardiectomy including the removal of the visceral pericardium is the remaining therapeutic option.
\end{abstract}

Keywords: pericarditis, etiology, constriction, hemodynamics of ECP, treatment

\section{Introduction}

Effusive-constrictive pericarditis (ECP) is a rare but increasingly recognized syndrome within the spectrum of pericardial diseases. In the first pericardial disease guidelines worldwide, ${ }^{1}$ its hemodynamics was defined as "no fall of pressures after pericardial drainage", and as one of the etiologies, tuberculous pericarditis was explicitly mentioned. In the update of the guidelines, ${ }^{2}$ the description of chronic permanent constrictive pericarditis was challenged by specific variants of constrictive syndromes such as transient constriction and effusive-constrictive forms.

\section{Basics}

The pericardium consists of 2 layers, a parietal and a visceral layer. The visceral pericardium is composed of mesothelial cells and adheres closely with the epicardium (Figure 1). ${ }^{3}$

The parietal pericardium is separated from the visceral pericardium by a small amount of fluid of 10-15 mL, which serves as a lubricant. Accumulation of fluid
Correspondence: Bernhard Maisch Feldbergstraße 45, 35043 Marburg,

Germany

Tel +49 642I 94690

Fax +496421946915

Email bermaisch@gmail.com
Journal of Vascular Diagnostics and Interventions 2018:6 7-14 


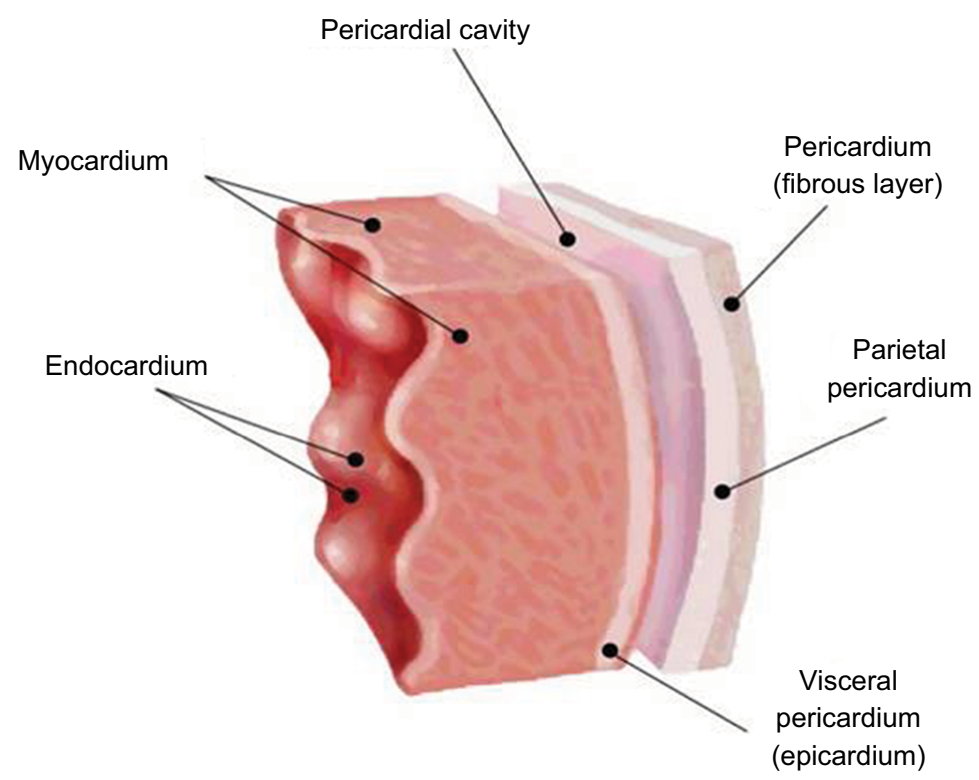

Figure I Cross-section of the pericardial and myocardial layers.

Note: Adapted from Maisch B. ESC-Webinar 2017: diagnosis and management of cardiac tamponade and constrictive pericarditis according to the ESC guidelines on pericardial disease. Available from: https://www.escardio.org/Education/E-Learning/Webinars/General-cardiology/diagnosis-and-management-of-constrictive-pericarditis. ${ }^{3}$

beyond this limit is termed pericardial effusion. ${ }^{4-6}$ As a general rule, a symptomatic pericardial effusion accessible for pericardiocentesis should be extensively evaluated to determine its etiology and hemodynamic significance and could be explored as the location for local treatment. ${ }^{7}$ For ECP, this principle should be applied. In ECP cases with effusions, which are too small to permit a safe access to the pericardium, endomyocardial biopsy may take the role of epi- and pericardial biopsy and pericardial fluid assessment.

\section{Epidemiology}

Effusive-constrictive variants of pericarditis were first described in the late 1960s. ${ }^{8}$ But it was Hancock who coined the definition of a constrictive physiology with a coexisting pericardial effusion ${ }^{9}$ and described it more precisely later. ${ }^{10}$ The starting point of a broader discussion was a Spanish series of 1184 pericarditis patients in whom the prevalence of ECP was $1.3 \% .{ }^{11}$ The original definition of ECP was made based on patients undergoing pericardiocentesis in tamponade, in whom after normalization of the pericardial pressure the right atrial pressure did not fall below $50 \%$ or $10 \mathrm{~mm} \mathrm{Hg}$. A French series of 141 patients with pericardial effusion reported an incidence of ECP in 2 of the patients (1.4\%). ${ }^{12}$ In American patients with chronic pericardial effusion requiring pericardiectomy, a prevalence of $4.3 \%{ }^{13}$ was reported. In an earlier series of patients with constrictive pericarditis, an incidence of $24 \%$ was reported. ${ }^{14}$ In our prospective German series of 259 consecutive patients undergoing pericardiocentesis, pericardioscopy and simultaneous intrapericardial and right atrial pressure monitoring, ECP was found by retrospective analysis in 9 patients with different etiologies of the pericardial disease (3.4\%). ${ }^{15}$

\section{Pathophysiology of ECP}

ECP is defined by concurrent pericardial effusion and pericardial constriction. Whereas in constrictive pericarditis the pericardial cavity is obliterated without any effusion, in patients with an ECP, the scarred pericardium not only constricts cardiac chambers but the additional pericardial fluid also increases intrapericardial pressure, which may mimic signs of cardiac tamponade. This pressure on cardiac chambers can persist even after the entire pericardial effusion is removed. The underlying pathogenetic process predominantly involves the visceral pericardium or epicardium. Its main pathogenetic mechanism is thought to be visceral pericardial constriction together with residual pericardial inflammation and effusion. The nature of visceral pericardial constriction was first studied on pericardiectomy, where both parietal and visceral pericardium demonstrated significant fibrosis with interspersed adhesions and fluids. ${ }^{10,11,16,17}$ Fibrosis and adhesions can also be appreciated by pericardioscopy in patients with persistently elevated right atrial pressures after pericardiocentesis. ${ }^{15,18}$

\section{Diagnosis}

\section{Clinical presentation}

Symptoms in patients with ECP are variable. Due to small residual pericardial effusions, the patients may have chest 
pain, fatigue and leg edema and, on inspection, they may even show signs of tamponade or transient constriction.

\section{Physical examination}

At bedside elevated jugular pressure similar to constrictive pericarditis is the hallmark of effusive-constrictive pericarditis even after pericardiocentesis. In patients with very little pericardial effusion, the jugular pressure is elevated disproportionate to the amount of pericardial fluid.

\section{Electrocardiography}

There are no pathognomonic electrocardiogram features in ECP. They may vary from ST-T segment changes to reduced QRS voltage. Heart rate is elevated.

\section{Heart catheterization}

A drop in the right atrial pressure below $10 \mathrm{~mm} \mathrm{Hg}$ or by more than $50 \%$ is diagnostic of ECP. Dip and plateau phenomena are common and are similar to patients with constriction (Figure 2).

\section{Echocardiography}

A comprehensive echocardiography with Doppler evaluation should take into consideration the criteria for constrictive disease. These are (1) respiration-related ventricular septal shift, (2) preserved or increased medial mitral e' velocity, (3) the prominent hepatic vein expiratory diastolic flow reversals, (4) annulus reverses and (5) annulus paradoxus.

In ECP, echo-Doppler can also show features of pericardial fibrosis (Horowitz F in TM-echocardiography) often combined with small effusions (Horowitz B or C1), particularly after pericardiocentesis. Rarely is epicarditis as prominent as in Figure 3.

In a group of patients with hemodynamically defined ECP of tuberculous origin, features in echocardiography with $81 \%$ sensitivity and $75 \%$ specificity were a respiratory variation of the mitral E wave or a respirophasic septal shift. The mitral $\mathrm{e}^{\prime}$ velocities and shorter mitral deceleration times were additional features that could distinguish ECP patients from those with "simple" pericardial effusion. ${ }^{19}$ By a subxyphoidal approach,

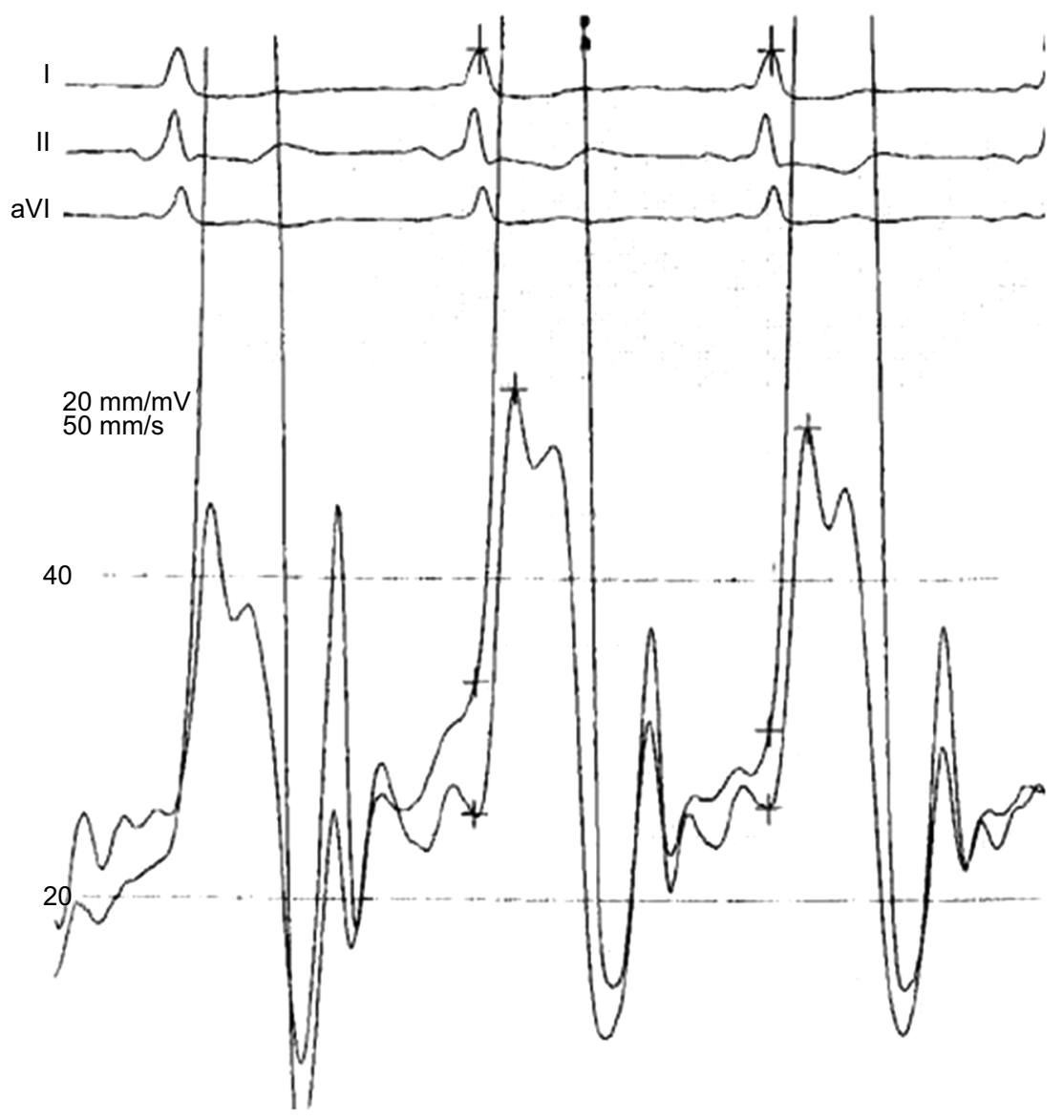

Figure 2 Dip-and-plateau phenomenon in the left and right ventricles of a patient with persisting signs of tamponade after pericardiocentesis. The persistance of an elevated end-diastolic pressure with the classic square-root sign of tamponade is diagnostic, although tamponade is no longer present. This is one of the characteristic hemodynamic features of ECP.

Abbreviation: ECP, effusive-constrictive pericarditis. 


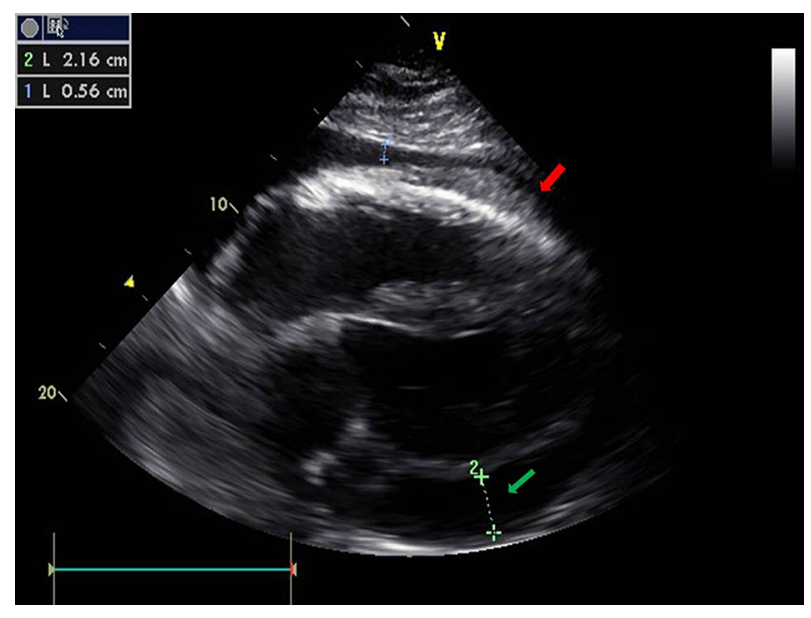

Figure 3 Epicardial inflammatory and fibrinous tissue on the epicardial side of the right ventricle (red arrow) in a 49-year-old patient with recurrence of a loculated pericardial effusion (green arrow). Most of the loculated effusion could be removed by pericardiocentesis, but signs of tamponade persisted due to epicarditis. The epicardial biopsy of this mass revealed fibrous tissue, fibrinogen and fat cells only. Neither microbial DNA or RNA by PCR nor malignancy could be detected.

dilatation of the inferior vena cava is a regular finding. By this approach, small pericardial effusion can also be best identified. A comparison of the echo-criteria of ECP with those of tamponade and restrictive cardiomyopathy is done by Maisch et al. ${ }^{7}$

It is still a matter of discussion, however, if echo-Doppler criteria alone show enough specificity and sensitivity for making a noninvasive diagnosis of ECP.

\section{Cardiac computed tomography and magnetic resonance imaging (MRI)}

Both methods can contribute to the assessment of the characteristic features of ECP: pericardial thickening ( $>4 \mathrm{~mm}$ ) and small residual effusion. Cardiac resonance imaging and MRI may also detect inflammation by late gadolinium enhancement (LGE) of the visceral and parietal pericardial layers and, in cases of perimyocarditis, in the adjacent cardiac tissue as in Figure 4. T2 imaging by MRI may be useful in distinguishing moving fluid from stationary fluid, for example, in loculated effusions or in ECP. In addition, RF tissue-tagging represents a unique method of detecting constriction, especially after pericardial effusion is removed or is very small and ECP is considered.

The case of a 34-year-old female half-marathon runner with recurrent pericarditis at the time of admission to our hospital may be of particular interest (Figure 4). The patient had had a previous episode of pericarditis which was treated with colchicine $2 \times 0.5 \mathrm{mg}$. She started with competitive sports again after 12 weeks of physical restraint despite the warning of her local cardiologist. At the time of admission to our hospital, she was having heart failure, New York Heart Association (NYHA) class III, and ejection fraction was

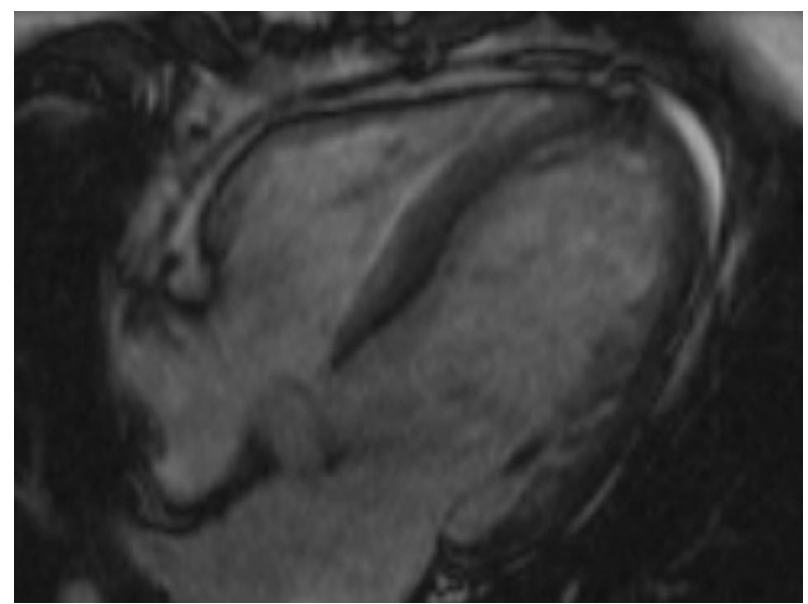

Figure 4 Cardiac MRI of a 34-year-old female half-marathon runner with recurrent pericarditis at the time of admission to our hospital.

Abbreviation: MRI, magnetic resonance imaging.

$36 \%$ by echocardiography with still normal left and right ventricular dimensions. LGE of myocardial and epicardial tissues at the lateral side of the right ventricle and a small pericardial effusion were the respective findings at MRI. She underwent biventricular endomyocardial biopsy, which demonstrated no viral DNA or RNA but inflammation in the right ventricle. Therefore, an autoreactive perimyocarditis was diagnosed. Together with the elevated left ventricular end-diastolic pressure and right ventricular end-diastolic pressure, a diagnosis of ECP with myocardial involvement was made and immunosuppressive treatment was initiated according to the European Study on Epidemiology and Treatment in Cardiomyopathy and Inflammation protocol. ${ }^{20}$ In detail, azathioprin $2 \mathrm{mg} / \mathrm{kg}$ body weight (BW)/day was administered for 4 weeks, and the maintenance dose for the residual 5 months was $0.85 \mathrm{mg} / \mathrm{kg} \mathrm{BW} /$ day; prednisolone $1.25 \mathrm{mg} / \mathrm{kg} \mathrm{BW} /$ day was administered for 4 weeks, and the maintenance dose for the residual time after tapering in 2.5 $\mathrm{mg}$ steps every 3rd day was $0.3 \mathrm{mg} / \mathrm{kg} \mathrm{BW} /$ day. In addition to heart failure medication (ramipril $5 \mathrm{mg}$, bisoprolol $5 \mathrm{mg}$ ), diuretics were administered whenever needed. After 6 months of treatment, the end-diastolic pressures had normalized and the small pericardial effusion and LGE had disappeared.

\section{Laboratory findings}

These should include the measurement of C-reactive protein as an inflammatory marker, and biomarkers of cardiac necrosis, for example, creatinine kinase muscle brain subtype and high-sensitivity troponins, which are elevated in almost all cases. Differential white blood cell count may show changes depending on the individual etiologies differing from patient to patient. 
Assessment of the underlying etiologies, particularly tuberculosis in the African countries, and determination of rheuma serology, for example, antinuclear antibodies, extractable nuclear antibodies and cardiac autoantibodies, antimyolemmal antibodies (AMLA) and antifibrillary antibodies (AFA), complete the clinical assessment and point to an autoreactive etiology. ${ }^{1,17,18,25}$

\section{Pericardioscopy}

In patients undergoing pericardiocentesis, in whom after evacuation of the pericardial fluid the criteria of ECP (normal intrapericardial pressure, but persistently elevated right atrial pressure) are present, pericardioscopy and targeted epi- and pericardial biopsy can be carried out in specialized centers to clarify the etiological process. The already inserted pigtail catheter in the pericardial sac is exchanged with a flexible endoscope via a guidewire. The pericardioscopic images in the white light mode or even better by the blue light mode can demonstrate both sides of the pericardial layers and the interspersing strand. They also show dark spots, which are non- fibrotic lesions from which epicardial biopsies can deliver the best results for an etiological diagnosis ${ }^{7,15}$ (Figure $5 \mathrm{~A}$ and $\mathrm{B}$ ). Figure $5 \mathrm{C}$ demonstrates her visceral pericardium with patchy fibrinous lesion at the moment when the biopsy was taken. Figure 5D demonstrates a larger adhesive fibrous zone and adjacent reddish area of inflamed epicardium.

\section{Cardiac biopsies and fluid analysis}

Epicardial and pericardial biopsies taken in the context of pericardiocentesis are the suitable diagnostic tool to exclude or prove a viral etiology by $\mathrm{PCR}^{3,15}$ and lead the way to corticoid and immunosuppressive treatment. ${ }^{18,19} \mathrm{~A}$ cardiac center performing pericardiocentesis and peri- and epicardial biopsies should have at least 2 institutional features: a pericardiologist experienced in biopsy performance and pericardiocentesis and a dedicated laboratory to perform histology, immunocytochemistry and molecular techniques such as PCR on cardiotropic agents in biopsy specimens. Malignant pericardial effusion can be diagnosed by cytology of the fluid and targeted biopsy under pericardioscopical
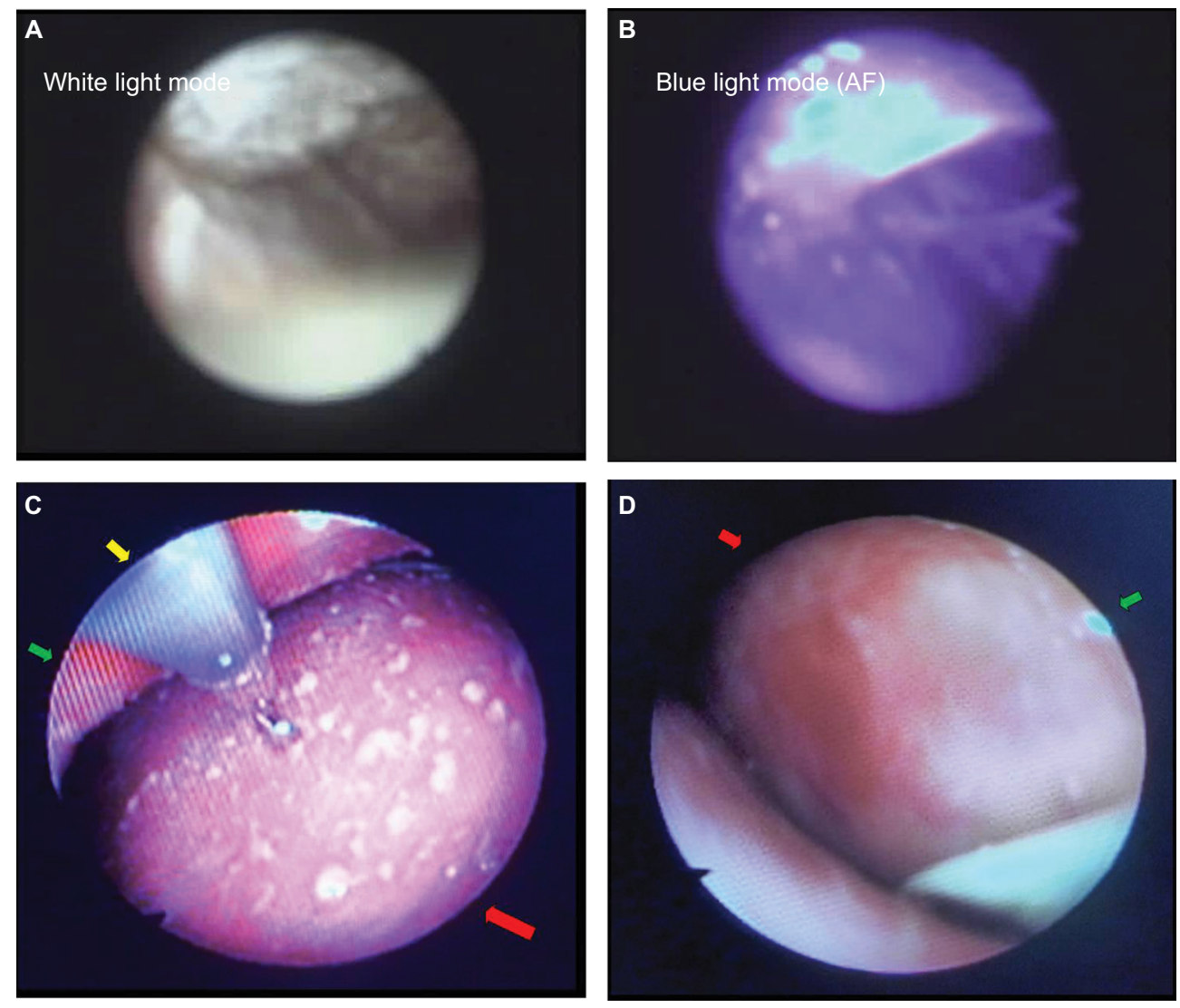

Figure 5 (A) Pericardioscopy: thickening of parietal and visceral pericardium with interspersed adhesions and some residual fluid (white light mode). (B) The interlinking adhesions (white strands) can be appreciated best in the blue light mode. The dark spots are areas of inflammation. They are the primary targets of epicardial biopsies to assess the underlying inflammation. (C) Epicarditis with small patchy fibrinous lesions. The biopsy was PCR negative for microbial DNA and RNA. Red arrow: inflamed visceral pericardium (epicardium), green arrow: parietal pericardium also with fibrinous lesions and yellow arrow: bioptome. (D) Epicarditis with a large red colored inflammatory lesion (red arrow) and an adhesive fibrous white area (green arrow). (A and B) Reprinted by permission from Springer Nature: Heart Fail Rev, Pericardioscopy and epi- and pericardial biopsy - a new window to the heart improving etiological diagnoses and permitting targeted intrapericardial therapy, Maisch B, Rupp H, Ristic A, Pankuweit S, 20I3. ${ }^{15}$ 
control. With these techniques, it is nowadays possible to avoid the virtually unhelpful diagnosis of "idiopathic" ECP. ${ }^{18}$

\section{Etiological factors in pericardial effusion and ECP}

The landmark contribution on the etiology of pericardial effusion of 259 patients undergoing pericardiocentis, pericardioscopy, biopsy and fluid analysis by $\mathrm{PCR}^{15}$ in our tertiary referral center demonstrated malignant disease in $28 \%$, autoreactive or lymphocytic inflammation in $35 \%$, viral etiology in $12 \%$, bacterial infection in $2 \%$, trauma in $15 \%$ and other or unknown causes in $8 \%$. (Table 1). In the viral group ( $\mathrm{n}=34$ ), Parvovirus B19 prevailed in $62 \%$, Epstein-Barr virus in 29\% and double infections of both in $3 \%{ }^{21}$ The database of these 2 contributions ${ }^{15,18,21}$ contained 9 patients with hemodynamics and features of ECP. Three of these 9 patients had malignant disease ( 2 bronchocarcinomas and 1 breast cancer), and 6 had a virus-negative epicardial biopsy with the demonstration of AMLA and AFA and were classified as nonviral autoreactive pericardial effusion. Since publications on ECP are rare and the availability of PCR is restricted to a few centers, previous publications have classified patients with ECP mostly as idiopathic. In contrast to Europe and the USA, tuberculous infection, often together with HIV, prevails in the etiology of pericardial effusion and in constrictive-effusive pericarditis. ${ }^{1,2,14,17}$ For comparison, the dominant etiologies in constrictive pericarditis in North America and Europe are idiopathic ECP, post-cardiac surgery and radiation therapy ${ }^{7}$.

After rinsing with at least $100 \mathrm{~mL}$ saline, $80 \mathrm{mg}$ gentamycin was administered intrapericardially in all patients immediately after pericardiocentesis. The pigtail catheter was left in place under sterile conditions. The patients were monitored in the intensive care unit under i.v. ampicillin prophylaxis. The results of histology, cytology and PCR were available after 24 hours to decide on further intrapericardial and other therapy.

\section{Treatment}

Treatment should be orientated to eliminate the underlying cause. Table 1 gives an overview of the intrapericardial treatment algorithm in ECP. The total cohort of all 259 patients with pericardial effusion undergoing pericardiocentesis and eventual intrapericardial therapy could be subdivided in $28 \%$ malignant, $35 \%$ autoreactive/lymphocytic, $12 \%$ viral, $2 \%$ bacterial, $15 \%$ traumatic, $8 \%$ other forms of effusions. ${ }^{15} \mathrm{In}$ neoplastic effusion, $30 \mathrm{mg} / \mathrm{m}^{2}$ cisplatin in $100 \mathrm{~mL}$ of $0.9 \%$ saline was given in a single slow injection through the intrapericardially located pigtail catheter.

In autoreactive or lymphocytic virus-negative pericarditis, either $300 \mathrm{mg} / \mathrm{m}^{2}$ or $600 \mathrm{mg} / \mathrm{m}^{2}$ triamcinolone was injected intrapericardially. The instillation of this crystalloid suspension stays active for 3-4 weeks. In cases with suspected additional myocardial involvement, oral azathioprine was added for 6 months at a dose of 75-100 mg. Colchicine 2-3 $\times 0.5 \mathrm{mg}$ was added for the next 6 months as additional oral medication. As suggested in the guidelines, ${ }^{1,2}$ aspirin 500 $1000 \mathrm{mg}$ every 8 hours, ibuprofen $600 \mathrm{mg}$ every 8 hours and indomethacin $25-50 \mathrm{mg}$ every 8 hours can be administered in addition as antiphlogistic and for pain therapy, if needed.

Experience in the combined treatment of HIV and tuberculosis is very limited in Europe and the USA. Tuberculous pericarditis is often combined with HIV infection and in this combination most prevalent in African countries. ${ }^{16,22}$ In tuberculous ECP, no intrapericardial treatment has yet been reported. The mainstay is long-term tuberculostatic medication. It may be combined with low-dose corticosteroids at the beginning to avoid constrictive disease.

In malignant disease showing features of ECP, treatment should be discussed with the oncologist, as in all patients with malignant effusions. We had excellent results in 3 patients with malignant ECP out of the cohort of 9 ECP patients, with intrapericardial cisplatin to avoid recurrence of tamponade. Our institution has reported the efficacy with a much larger cohort of patients with malignant pericardial effusion. ${ }^{23,24}$

Table I Etiologies of pericardial effusion with ECP in a German tertiary referral center

\begin{tabular}{lllll}
\hline Aetiology & $\begin{array}{l}\text { Effusions (n=259), } \\
\text { \% positive }\end{array}$ & $\begin{array}{l}\text { ECP (n=9), } \\
\text { \% positive }\end{array}$ & Intrapericardial treatment & Other \\
\hline Malignant & 28 & 33,3 & Gentamycin + cisplatin & Systemic tumor therapy \\
Autoreactive/lymphocytic & 35 & 66,7 & Gentamycin + triamcinolone & Azathioprin + colchicine \\
Viral (Parvovirus BI9, EBV) & 12 & 0 & Gentamycin & i.v. immunoglobulins (Pentaglobinß) \\
Bacterial & 2 & 0 & Gentamycin & i.v. antibiotics, cardiac surgery \\
Trauma & 15 & 0 & Gentamycin & Colchicine \\
Other/idiopathic & 8 & 0 & &
\end{tabular}

Notes: Data from Maisch et al. ${ }^{15}$

Abbreviations: ECP, effusive-constrictive pericarditis; EPV, Epstein-Barr virus. 
Algorithm in ECP

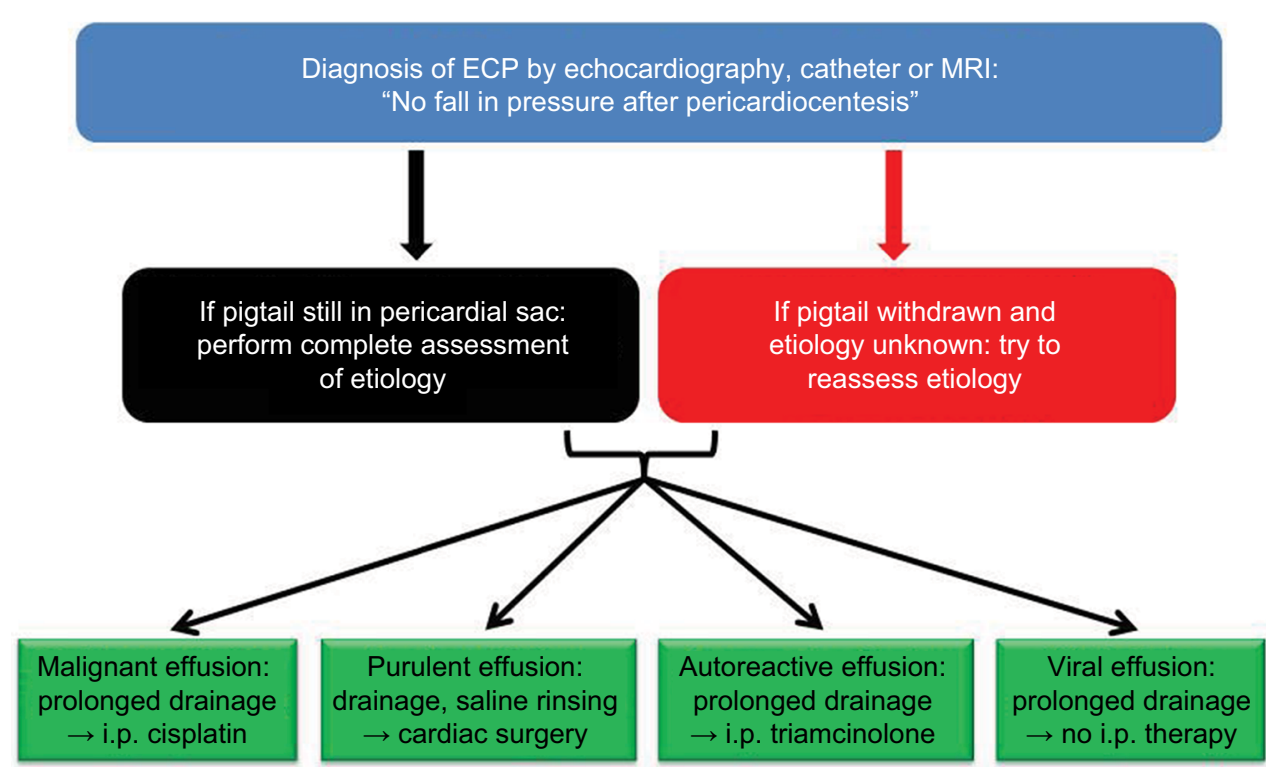

Figure 6 Diagnostic and therapeutic algorithm in ECP.

Abbreviations: $E C P$, effusive-constrictive pericarditis; $M R I$, magnetic resonance imaging.

If a viral etiology is excluded by cardiac biopsies, intrapericardial treatment with triamcinolone avoids many side effects of systemic corticoid treatment in these patients. ${ }^{25} \mathrm{~A}$ crystalloid suspension of triamcinolone administered intrapericardially and left there will exert its effect for at least 3 weeks. ${ }^{7,18,25}$ We now combine intrapericardial triamcinolone with a daily oral dose of $100 \mathrm{mg}$ azathioprine for at least 6 months in patients with autoreactive pericardial effusion with or without additional myocardial involvement. An additional treatment with colchicine $0.5 \mathrm{mg}$ dispert 2-3 tablets per day was well tolerated in the the subgroup of 6 ECP patients with autoreactive effusions. In summary, immunosuppression in the cohort of 6 virus-negative, nonmalignant ECP patients eliminated the small residual effusion and the constrictiveeffusive hemodynamic pattern effectively.

If medical treatment, oral or intrapericardial, should fail, complete pericardiectomy by an experienced cardiac surgeon remains the final option. ${ }^{26,27}$

\section{Overlap to similar syndromes}

ECP has obvious similarities with transient constrictive pericarditis ${ }^{11}$ and occult constrictive pericarditis. ${ }^{28}$ In patients with predominant myocardial inflammation, as the one shown in Figure 4, restrictive and constrictive features may overlap.

\section{Take-home message}

ECP is a part of the entity of inflammatory pericardial diseases. Its definition is, as of today, merely by its hemodynamic status as "no fall of pressures after pericardial drainage". The algorithm to follow is outlined in Figure 6.

Our most important perspective is to clarify the underlying etiology of ECP. This should be much more in the focus of cardiologists and internists since only causative treatment eliminates or ameliorates the disease, which may have ECP as its hemodynamic consequence.

\section{Disclosure}

The author reports no conflicts of interest in this work.

\section{References}

1. Maisch B, Seferovic PM, Ristic AD, et al; Task Force on the Diagnosis and Management of Pricardial Diseases of the European Society of Cardiology. Guidelines on the diagnosis and management of pericardial diseases. Executive summary; the Task Force on the Diagnosis and Management of Pericardial Diseases of the European Society of Cardiology. Eur Heart J. 2004;25(7):587-610.

2. Adler Y, Charron P, Imazio M, et al. 2015 ESC guidelines for the diagnosis and management of pericardial diseases. Eur Heart $J$. 2015;36(42):2921-2961.

3. Maisch B. ESC-Webinar 2017: diagnosis and management of cardiac tamponade and constrictive pericarditis according to the ESC guidelines on pericardial disease. Available from: https://www. escardio.org/Education/E-Learning/Webinars/General-cardiology/ diagnosis-and-management-of-constrictive-pericarditis .

4. Goldstein JA. Cardiac tamponade, constrictive pericarditis and restrictive cardiomyopathy. Curr Probl Cardiol. 2004;29(9):503-567.

5. Spodick DH. The Pericardium: A Comprehensive Textbook. New York, NY: Marcel Dekker; 1997.

6. Shabetai R. Pericardial Disease: Etiology, Pathophysiology, Clinical Recognition, and Treatment. New York, NY: Churchill Livingstone; 1995.

7. Maisch B, Ristic AD, Seferovic P, Tsang TSM. Interventional Pericardiology. Heidelberg: Springer Medizin; 2011. 
8. Spodick DH, Kumar S. Subacute constrictive pericarditis with cardiac tamponade. Dis Chest. 1968;54(1):62-66.

9. Hancock EW. Subacute effusive-constrictive pericarditis. Circulation. 1971;43(2):183-192.

10. Hancock EW. A clearer view of effusive-constrictive pericarditis. $N E n g l$ J Med. 2004;350(5):435-437.

11. Sagrista-Sauleda J, Angel J, SanchezA, Permanyer-Miralda G, Soler-Soler J. Effusive-constrictive pericarditis. N Engl J Med. 2004;350(5):469-475.

12. Nugue O, Millaire A, Porte H, et al. Pericardioscopy in the etiologic diagnosis of pericardial effusion in 141 consecutive patients. Circulation. 1996;94(7):1635-1641.

13. Tsang TS, Barnes ME, Gersh BJ, Bailey KR, Seward JB. Outcomes of clinically significant idiopathic pericardial effusion requiring intervention. Am J Cardiol. 2003;91(6):704-707.

14. Cameron J, Oesterle SN, Baldwin JC, Hancock EW. The etiologic spectrum of constrictive pericarditis. Am Heart J. 1987;113(2 Pt 1):354-360.

15. Maisch B, Rupp H, Ristic A, Pankuweit S. Pericardioscopy and epi- and pericardial biopsy - a new window to the heart improving etiological diagnoses and permitting targeted intrapericardial therapy. Heart Fail Rev. 2013;18(3):317-328.

16. Syed FF, Ntsekhe M, Mayosi BM, Oh JK. Effusive-constrictive pericarditis. Heart Fail Rev. 2013;18(3):277-287.

17. Miranda WR, Oh JK. Effusive-constrictive pericarditis. Cardiol Clin. 2017;35(4):551-558.

18. Maisch B, Ristic AD, Pankuweit S, Seferovic P. Percutaneous therapy in pericardial diseases. Cardiol Clin. 2017;35(4):567-588.

19. Van der Bijl P, Herbst P, Doubell AF. Redefining effusive-constrictive pericarditis with echocardiography. $J$ Cardiovasc Utrasound. 2016;24(4):317-323.
20. Hufnagel G, Pankuweit S, Richter A, Schönian U, Maisch B. The European Study of Epidemiology and Treatment of Cardiac Inflammatory Diseases (ESETCID). First epidemiological results. Herz. 2000;25(3):279-285.

21. Pankuweit S, Stein A, Karatolios K, Richter A, Ruppert V, Maisch B. Viral genomes in the pericardial fluid and in peri- and epicardial biopsies from a German cohort of patients with large to moderate pericardial effusions. Heart Fail Rev. 2013;18(3):329-336.

22. Ntsekhe M, Mayosi BM. Tuberculous pericarditis with and without HIV. Heart Fail Rev. 2013;18(3):367-373.

23. Maisch B, Ristic AD, Pankuweit S, Neubauer A, Moll R. Neoplastic pericardial effusion: efficacy and safety of intrapericardial treatment with cisplatin. Eur Heart J. 2002;23(20):1625-1631.

24. Maisch B, Ristic A, Pankuweit S. Evaluation and management of pericardial effusion in patients with neoplastic disease. Prog Cardiovasc Dis. 2010;53(2):157-163.

25. Maisch B, Ristic AD, Pankuweit S. Intrapericardial treatment of autoreactive pericardial effusion with triamcinolone: the way to avoid side effects of systemic corticosteroid therapy. Eur Heart $J$. 2002;23:1503-1508.

26. Cho YH, Schaff HV. Surgery for pericardial disease. Heart Fail Rev. 2013;18(3):375-387.

27. Bicer M, Oezdemir B, Kan I, Yüksel A, Tok M, Şenkaya I. Long-term outcomes of pericardiectomy for constrictive pericarditis. J Cardiothorac Surg. 2015;10:177-182.

28. Bush CA, Stang JM, Wooley CF, Kilman JW. Occult constrictive pericardial disease. Diagnosis by rapid volume expansion and correction by pericardiectomy. Circulation. 1977;56(6): 924-930.
Journal of Vascular Diagnostics and Interventions

\section{Publish your work in this journal}

The Journal of Vascular Diagnostics and Interventions is an international, peer-reviewed journal of diagnostics, focusing on non invasive vascular investigation methods involved in the evaluation of vascular diseases. The journal is committed to the rapid publication in the fields of vascular diseases. Original research, review, case reports, expert

\section{Dovepress}

opinion and commentaries are all considered for publication. The manuscript management system is completely online and includes a very quick and fair peer-review system, which is all easy to use. Visit $\mathrm{http}: / /$ www.dovepress.com/testimonials.php to read real quotes from published authors. 